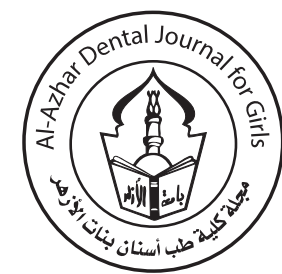

\title{
Effectiveness of Chitosan as Final Irrigating Solution on Bond Strength of Resin Based Sealer to Radicular Dentin
}

\author{
Sarah S. Hashem ${ }^{1}$, Mervat I. Fawzy ${ }^{2}$, Hagar A. Bastawy ${ }^{3}$
}

Codex : 19/1904

azhardentj@azhar.edu.eg

http://adjg.journals.ekb.eg

DOI: $10.21608 /$ adjg.2019.5953.1034

\section{KEYWORDS}

Chitosan, Irrigating solution, AH plus sealer, Bond strength.

\begin{abstract}
Purpose: This study was designed to assess the impact of Chitosan-acetate as final root canal irrigant on bond strength of resin-based sealer to radicular dentin. Materials and Methods: Forty extracted human lower premolars with single roots and canals were prepared with ProTaper Universal rotary files till size F4. The samples were dispensed into 3 experimental groups according to the final rinse used and control group (10 samples each); Group I: irrigated with $0.2 \%$ chitosan acetate, Group II: $17 \%$ EDTA, Group III: distilled water and control group (IV): saline. The samples were obturated by cold lateral compaction technique using AH plus sealer. Samples were sectioned into three sections (each $2 \mathrm{~mm}$-thickness) from coronal, middle and apical thirds of each sample. The push out bond strength test was performed using universal testing machine. Three sections from each group were split longitudinally in buccolingual direction where the sealer/dentin interface and the sealer penetration inside the dentinal tubules was evaluated using scanning electron microscope (SEM). Results: Regarding the coronal and middle levels, there was no statistical significant difference among the tested groups. However, at the apical level, there was a statistical significant difference among the tested groups. SEM observation revealed minimal sealer penetration in group I at the apical level, while in group II, there was minimal sealer penetration at the coronal and middle levels. Regarding group III, there was minimal sealer penetration at middle level, while in control group (IV), there was no sealer penetration. Conclusion: The effect of $0.2 \%$ Chitosan acetate on the push out bond strength of $\mathrm{AH}$ Plus sealer was comparable to that of $17 \%$ EDTA when used as a final rinse. AH Plus sealer showed better adhesion to the dentin in the presence of smear layer especially at the apical third.
\end{abstract}

- Paper extracted from Master thesis titled "Effectiveness of Chitosan as Final Irrigating Solution on Bond Strength of Resin Based Sealer to Radicular Dentin".

1. Dentist at Al-Zahraa University Hospital.

2. Professor of Endodontics, Endodontic Department, Faculty of Dental Medicine for Girls, Al-Azhar University.

3. Associate Professor of Endodontics, Endodontic Department, Faculty of Dental Medicine for Girls, Al -Azhar University. 


\section{INTRODUCTION}

Perfect endodontic treatment relay on complete cleaning \& shaping of radicular pulp space and achive three-dimensional filling with biocompatible, and dimensionally stable filling material. Using of nickel-titanium files alone in root canal preparation is insufficient as more than $35 \%$ of the root canal's surface can be left un-instrumented after conventional root canal treatment ${ }^{(1)}$. In order to clean these un-instrumented surfaces, it is necessary to irrigate the root canal abundantly for killing microorganisms, lubrication, removing debris and smear layer created by cleaning $\&$ shaping of the radicular pulp space ${ }^{(2)}$.

Sodium hypochlorite $(\mathrm{NaOCl})$ solution is the gold standard because of its exceptional qualities as an antiseptic and its tissue dissolving effects. However, it is active only against organic part of smear layer, so other substances must be used to remove the inorganic material. Therefore, chelating compounds like ethylenediamine tetraacetic acid (EDTA) and citric acid (CA) were recommended as adjuvant in root canal treatment ${ }^{(3)}$.

Irrigation solutions at our disposable have their share of limitations, therefore natural products are widespread nowadays because of their antimicrobial activity, anti-inflammatory, biocompatibility and anti-oxidant properties ${ }^{(4)}$. Chitosan is a natural polysaccharide, that received more attention in dental research due to its biocompatibility, biodegradability and non-toxicity. It was used extensively in various industrial fields because of its high chelating capability for different metal ions in acidic environment ${ }^{(5)}$. Chitosan is obtained from chitin through deacetylation process, this material found in shrimp shells and crabs and became ecologically exciting for various applications due to its plenty in nature and reduced costs of production ${ }^{(6)}$.

A preliminary study evaluated the chelating properties of chitosan as a (natural polymer) in comparison with other irrigating solutions on the middle third of prepared root canal using SEM re- vealed that $2 \%$ chitosan acetate effectively removed the smear layer as 17\% EDTA and using 2\% chitosan acetate followed by $\mathrm{NaOCl}$ produced a clean surface. These results suggested that chitosan is a promising chelating agent ${ }^{(7)}$. The impact of chitosan at various concentrations on dentin structure and on smear layer elimination was assessed. The results revealed that using $0.2 \%$ chitosan for $3 \mathrm{~min}$ was effective in eliminating the smear layer and resulting in slight dentinal erosion ${ }^{(8)}$. A recent study compared the smear layer removal from radicular dentin subjected to two root canal irrigants; $17 \%$ EDTA and $0.2 \%$ Chitosan using SEM. The results revealed that $0.2 \%$ chitosan eliminate smear layer more efficient than 17\% EDTA at the apical level of the root canal ${ }^{(9)}$.

Sealer is required to bond gutta-percha, which is the most popular root canal filling material to the radicular dentin as it is not able to adhere to radicular dentin alone ${ }^{(10)}$. The strength of the bond received more attention with the evolution of resin-based sealers, the possibility of producing a 'monoblock' of core material and sealer which also bonds to the radicular dentin has inserted the chance of strengthening the endodontically treated teeth. AH Plus sealer (epoxy resins-based sealer) has very good physical properties, small expansion, long working time, excellent apical sealing, adhesion to the tooth structure and adequate biological performance. Because of its desirable properties, it is widely used in the obturation of root canals ${ }^{(11)}$.

The impact of removing smear layer by various types of irrigating solutions on the bond strength of sealer to radicular dentin subjected to several studies. In some studies, improvement in the sealers' bond strength was reported when the smear layer was removed. However, other studies showed no significant difference or even higher values when the smear layer was present. A previous study revealed that the bond strength of AH plus sealer to radicular dentin improved upon elimination of smear layer by using maleic acid, EDTA, smear clear and CA as final rinses compared to the saline group ${ }^{(12)}$. Also, 
the impact of 17\% EDTA,0.2\% chitosan, QMix and distilled water as final irrigating solutions on bond strength of AH Plus, MTA Fillapex and Total Fill BC sealers to radicular dentin was assessed. The results revealed that the bond strength of all tested sealers decreased when irrigated with distilled water ${ }^{(13)}$. A recent study revealed that the greatest push out bond strength was noticed in samples irrigated with $\mathrm{NaOCl}$ alone (presence of smear layer) which was different from those samples irrigated with $\mathrm{NaOCl}$ + EDTA (absence of smear layer), regardless of the sealer type (AH plus or EndoRez) ${ }^{(14)}$. Another study assessed the impact of removing smear layer on the bonding of two resin based endodontic sealers ( $\mathrm{AH}$ 26 and $\mathrm{AH}$ plus). It was concluded that bonding of AH-26 to radicular dentin improved after treatment with 17\% EDTA. Conversely bonding of AH Plus sealer decreased following the treatment with $17 \%$ $\operatorname{EDTA}^{(15)}$.

Different methods were used for assessing the strength of sealers' bond to dentin, such as push-out bond strength test, shear bond test and tensile test. The push-out test showed several advantages, including the placement of sealer directly to radicular dentin, rather than sealer placement on flat coronal dentin discs, which shows a various order of dentinal tubules ${ }^{(16)}$. Moreover, it provides more accurate bond strength assessment than the traditional shear test as in the push-out test, fracture happens parallel to the dentin-bonding interface, which render it a real shear test for parallel-sided specimens (13). Therefore, the aim of this study was directed to assessed the impact of chitosan-acetate as final rinse on bond strength of resin-based sealer to radicular dentin using push out test.

\section{MATERIALS AND METHODS}

\section{Teeth selection and preparation}

Forty extracted human lower premolars with completely formed single roots and single canals were selected. All teeth were rinsed under tap water to eliminate blood, tissues and debris and kept in distilled water until use. Each tooth was decapitated at the cemento-enamel junction by using diamond disc under continuous water cooling and the root length was standardized to be $16 \mathrm{~mm}$. Sticky wax was used to close root apices in order to mimic closed-end model. K-files size \#10 were used to achieve canal patency and the working length was measured by subtracting $1 \mathrm{~mm}$ from length when the file tip observed at the apical foramina.

Preparation of root canals performed by using ProTaper Universal rotary NiTi files (Dentsply, Maillfer, Switzerland) in crown-down manner. The files were attached to a handpiece which was powered by an electric motor; at a rotational speed of $300 \mathrm{rpm}$ and a torque-control that was set according to manufacturer instructions for each file used. A set consisting of seven instruments was used, three shaping files (SX, S1 and S2) for preparation of coronal 2/3 and four finishing files (F1, F2, F3 and F4) for preparation of apical third used according to the instructions of manufacturer. Freshly prepared $2.6 \%$ $\mathrm{NaOCl}$ solution $(2 \mathrm{ml})$ was used to irrigate root canal for 1 minute after the use of each instrument, dispensed through a 31 gauge side vent irrigating needle that was inserted deeply into the root canal with no binding. In the control group, after each instrument use, irrigation was done with sterile saline.

\section{Preparation of chitosan solution}

Preparation of $0.2 \%$ chitosan acetate solutions was performed using $0.2 \mathrm{~g}$ chitosan powder (Sigma Co., Egypt), diluted in $100 \mathrm{ml}$ of $1 \%$ acetic acid, and a magnetic stirrer was used to stir the mixture for $2 h^{(8)}$. The prepared solution was saved in the refrigerator and used within two weeks after preparation $^{(17)}$.

\section{Samples grouping}

After preparation of root canal, the samples were distributed through 3 experimental groups (I, II, III) depending on the final rinse used and control group (IV) (10 samples each). Group I: irrigated 
with $0.2 \%$ chitosan acetate. Group II: $17 \%$ EDTA (Prevest Denpro limited, Digiana, Jammu, India). Group III: distilled water. Group IV (control group): sterile saline, each group was irrigated using $5 \mathrm{ml}$ of the irrigant for $3 \mathrm{~min}{ }^{(8)}$, then all samples were received $5 \mathrm{ml}$ of distilled water and dried by utilizing paper points.

\section{Samples obturation}

Obturation of samples was done by using ProTaper gutta-percha cones (\#F4) and AH Plus sealer (Dentsply De Trey Gmbh,Konstanz, Germany) which was mixed following the instructions of the manufacturer. The walls of the canal were coated with the sealer where, it was added on the master cone which moved vertically up and down inside the canal to ensure full coating of the canal wall by the sealer. Filling of root canal was completed by cold lateral condensation using \# 30 finger spreader size \# 30 and adding accessory cones (\# 25, .02). Then a hot instrument was utilized to get rid of the excessed gutta-percha and the access cavity was closed using temporary filling. Storage of samples at $37^{\circ} \mathrm{C}$ in $100 \%$ humidity for 48 hours was done to assure full setting of the sealer.

\section{Samples preparation for push-out bond strength testing:}

Isomet 4000 microsaw (Buehler, USA) was used to section the samples perpendicular to the root's long axis under constant water coolant, where three $2 \mathrm{~mm}$-thickness sections were gained from coronal, middle and apical levels of each sample. Indelible marker was used to mark the apical surface of each section, then the sections were kept in $100 \%$ humidity till testing.

Cylindrical stainless-steel plunger with diameter $1,0.7$ and $0.5 \mathrm{~mm}$ corresponding to coronal, middle and apical sections respectively used to load root filling of each section. The tip of the plunger was adjusted to cover the root filling material without contacting the canal wall. The plunger was connected to a universal testing machine (Instron, Norwood,
MA, USA) and the applied load was directed from apical aspect to coronal aspect in order to avoid any impediments during the push-out testing because of tapering of the root canal. A cross-head speed of $0.5 \mathrm{~mm} / \mathrm{min}$ was conducted until debonding happen and the maximum load conducted for debonding was measured in Newton $(\mathrm{N})$.

The following equation was used to calculate the value of push out bond strength in megapascal (MPa):

Push-out bond strength $(\mathrm{MPa})=$

Maximum load $(\mathrm{N})$

Adhesion area of root canal filling $\left(\mathrm{mm}^{2}\right)$

\section{Area of root canal filling $\left(\mathrm{mm}^{2}\right)=$}

(Circumference of coronal aspect + circumference of apical aspect)

2

$\mathrm{x}$ thickness of the section

$(2 \mathrm{~mm})$

\section{SEM evaluation:}

After the push-out test, one selected sample (3 sections) from each group were grooved longitudinally in buccolingual direction without reaching the internal portion of the canal and sectioned with sharp chisel. SEM was used to examine the sections which were coated with gold and mounted on an aluminum stub. Evaluation of the sealer/dentin interface and sealer penetration inside the dentinal tubules was done by taking photomicrographs under X 2000 magnification.

\section{Statistical analysis:}

Tests of normality (Kolmogorov-Smirnov and Shapiro-Wilk tests) were used to explore the numerical data for normality in addition to checking the distribution of data. Presentation of data as mean and standard deviation (SD) was performed. KruskalWallis test was used to compare between different groups in case of non-parametric data. Friedman's test was utilized to compare between different root levels. For pair-wise comparisons Dunn's test was 
used. The significance level was set at $\mathrm{P} \leq 0.05$. IBM SPSS Statistics Version 20 for Windows was utilized to carry out statistical analysis.

\section{RESULTS}

\section{I-Push out bond strength results:}

\section{Comparison of push out bond strength among the tested groups at each level:}

No statistical significant difference was found in the mean push out bond strength between the tested irrigants at coronal and middle levels. However, group III $(\mathrm{NaOCl})$ showed the highest value with a significant difference from the control group $(\mathrm{P} \leq$ $0.05)$ at apical level. Whereas, there was no statistical significant difference between $0.2 \%$ chitosan and 17\% EDTA groups. (Table 1) (Figure 1)

\section{Comparison of push out bond strength among root levels within each group:}

Among all the groups there was no statistical significant difference in the mean push out bond strength between the root levels within each group $(\mathrm{P}>0.05)$. (Table 1) (Figure 2)

Table (1): Descriptive analysis of the push out bond strength value and standard deviation at the coronal, middle and apical root canal levels of all tested groups.

\begin{tabular}{|c|c|c|c|c|c|c|c|c|c|}
\hline \multirow{2}{*}{ Root level } & \multicolumn{2}{|c|}{$\begin{array}{c}\text { Group I } \\
(0.2 \% \text { Chitosan })\end{array}$} & \multicolumn{2}{|c|}{$\begin{array}{c}\text { Group II } \\
\text { (17\% EDTA) }\end{array}$} & \multicolumn{2}{|c|}{$\begin{array}{l}\text { Group III } \\
\text { (NaOCl) }\end{array}$} & \multicolumn{2}{|c|}{$\begin{array}{c}\text { Group IV } \\
\text { (Saline) }\end{array}$} & \multirow{2}{*}{$P$-value } \\
\hline & Mean & SD & Mean & SD & Mean & SD & Mean & SD & \\
\hline Coronal & 5.33 & 1.90 & 5.76 & 1.49 & 5.71 & 1.88 & 4.39 & 1.29 & 0.196 \\
\hline Middle & 5.83 & 1.79 & 6.38 & 1.50 & 6.51 & 1.10 & 4.93 & 1.32 & 0.085 \\
\hline Apical & $5.55^{\mathrm{AB}}$ & 1.61 & $5.77^{\mathrm{AB}}$ & 1.55 & $7.11^{\mathrm{A}}$ & 1.77 & $4.27^{\mathrm{B}}$ & 1.70 & $0.015^{*}$ \\
\hline Total & $5.57 \mathrm{AB}$ & 1.13 & $5.97 \mathrm{AB}$ & 0.85 & $6.44^{\mathrm{A}}$ & 0.84 & $4.53^{\text {в }}$ & 0.86 & $0.003^{*}$ \\
\hline$P$-value & \multicolumn{2}{|c|}{0.301} & \multicolumn{2}{|c|}{0.497} & \multicolumn{2}{|c|}{0.497} & \multicolumn{2}{|c|}{0.905} & \\
\hline
\end{tabular}

*:Significant at $P \leq 0.05$, Different superscripts in the same row indicate statistically significant difference.

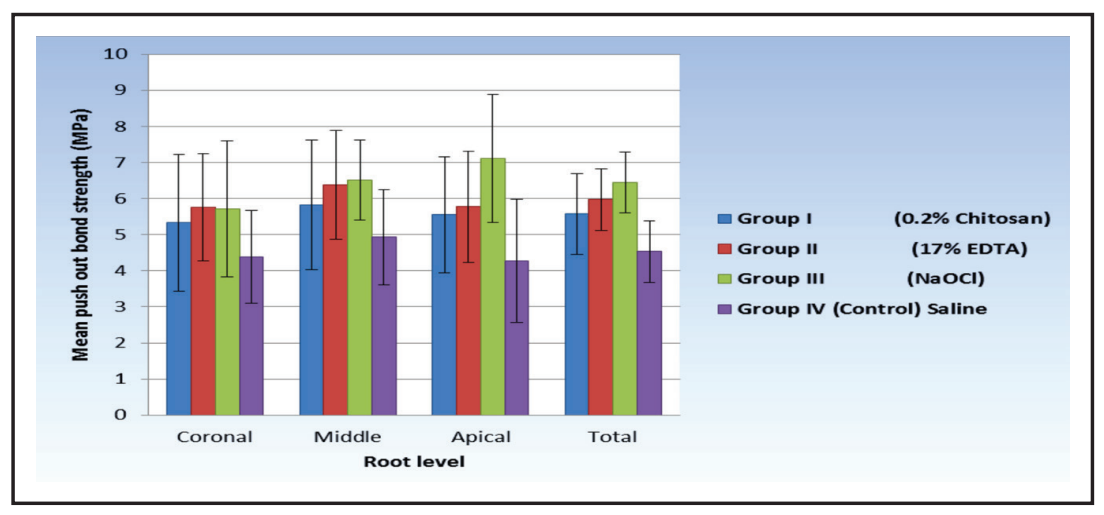

Figure (1): A bar chart comparing the mean push out bond strength and standard deviation among $0.2 \%$ Chitosan, $17 \%$ EDTA, $\mathrm{NaOCl}$ and saline (control) at the coronal, middle and apical root canal levels. 




Figure (2): A bar chart comparing the mean push out bond strength value and standard deviation at the coronal, middle and apical root canal levels within each tested group.

\section{Overall comparison between groups:}

Group III $(\mathrm{NaOCl})$ revealed the greatest mean push out bond strength with a statistical significant difference from the control group $(\mathrm{P} \leq 0.05)$. However, there was no significant difference between $0.2 \%$ chitosan and $17 \%$ EDTA groups. (Table 1, Figure 1)

\section{II- Scanning electron microscopic (SEM) results:}

A layer of $\mathrm{AH}$ plus sealer with filler particles embedded in the resin matrix was coating the canal walls in all groups.

In group I ( $0.2 \%$ Chitosan): Tracing the sealer/ dentin interface revealed that there was no resin tags packed inside the dentinal tubules at coronal and middle levels. However, there was minimal penetra- tion of the sealer at apical level. (Figure 3)

In group II (17\% EDTA): Tracing the sealer/ dentin interface revealed that there was minimal sealer penetration into at coronal and middle levels. However, no sealer penetration was noticed at apical level. (Figure 3)

In group III ( $\mathrm{NaOCl})$ : Tracing the sealer/ dentin interface revealed that there was minimal sealer penetration at the middle level. However, no sealer penetration noticed at coronal and apical levels. (Figure 3)

In group IV (control group) (saline): Tracing the sealer/ dentin interface revealed absence of sealer penetration at all levels.

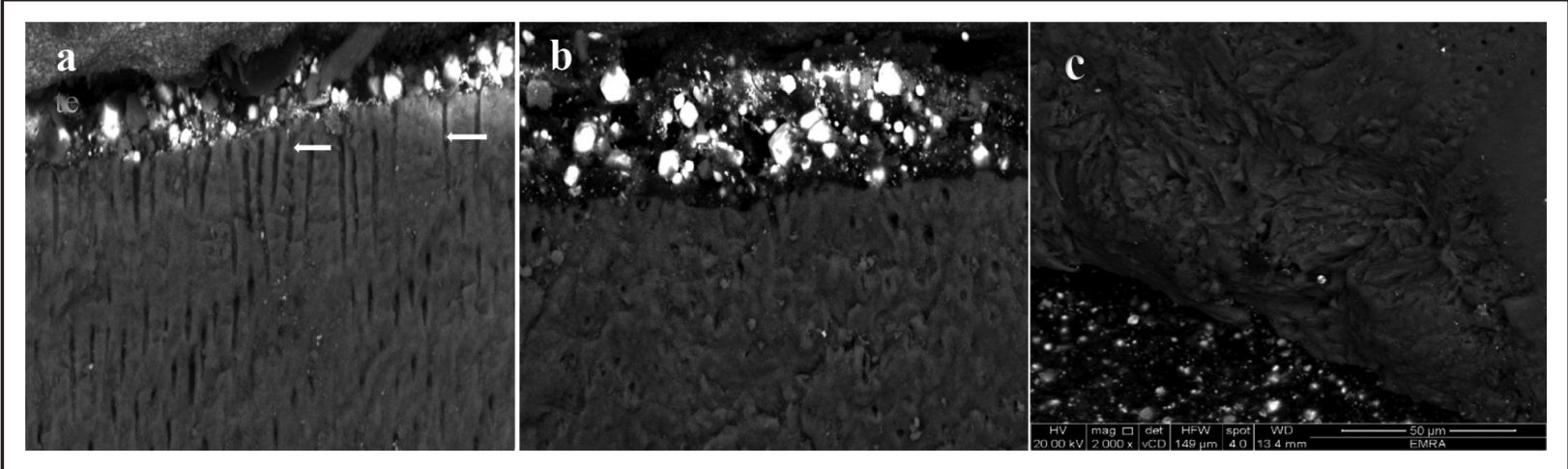

Figure (3): Scanning photomicrographs of sealer/dentin interface at the apical levels of root canals rinsed with; a) $0.2 \%$ chitosan showing minimal penetration of the sealer into the dentinal tubules, b) 17\%EDTA and c) NaOCl showing no sealer penetration into the dentinal tubules (X2000). 


\section{DISCUSSION}

Ideal endodontic treatment relay on different factors such as complete chemo-mechanical preparation of root canal and three-dimensional obturation with dimensionally stable and biocompatible filling material ${ }^{(18)}$. Gutta-percha that is used as a root canal filling material is not able to bond to the radicular dentin alone, thus a sealer is needed to achieve bonding between the gutta-percha and dentin to obtain a fluid tight seal. The adhesion of sealers to radicular dentin was improved by different methods, removing the smear layer is one of these methods. Until now no irrigating solution can act on the inorganic and organic constituents of the smear layer. For efficient removal of smear layer, a combination of $\mathrm{NaOCl}$ and EDTA has been recommended ${ }^{(19)}$. However, this combined irrigation regimen causes erosion to radicular dentin ${ }^{(20)}$.

Searching for chelating agent that is more efficient and biocompatible than EDTA has resulted in various solutions being researched over the last few years. Natural irrigants become more common nowadays because of their biocompatibility, high antimicrobial activity and anti-inflammatory (21). Therefore, this study was designed to assess the impact of chitosan-acetate as a final rinse on bond strength of resin-based sealer to radicular dentin.

In this study, the obtained results revealed that the highest mean push out bond strength was recorded at the middle level compared to the coronal and apical levels in samples treated with $0.2 \%$ chitosan acetate and 17\% EDTA (group I \& II respectively). However, no statistical significant difference was noticed in the mean push out bond strength among the root levels. This could be attributed to the fact that the diameter and the density of dentinal tubules significantly reduced from the coronal to apical direction. Thus, the diameter and number of the tubules at middle level are less than that at coronal level with more intertubular dentin which contains collagen fibers to which AH Plus sealer bond. The bonding of AH Plus sealer to dentin relays on covalent bonds formed between exposed amine groups on collagen and the open epoxy rings in the sealer, rather than micromechanical retention through penetration of the resin tags into the tubules ${ }^{(22,23)}$.

Regarding SEM observation of sealer/ dentin interface of tested samples which were treated with $0.2 \%$ chitosan acetate (group I) revealed that there was no penetration of sealer into dentinal tubules, however, in group II (17\% EDTA), there was minimal sealer penetration. This may be attributed to the viscosity of EDTA which was lower than that of $0.2 \%$ chitosan, so $17 \%$ EDTA could penetrate into dentinal tubules removing smear plugs, and thus permitting the penetration of $\mathrm{AH}$ plus sealer. This finding was in consent with a previous study which reported that there was little penetration of $\mathrm{AH}$ plus sealer after using $17 \%$ EDTA as a final rinse ${ }^{(23)}$.

The lower mean push out bond strength that was recorded at the apical level in samples treated with $0.2 \%$ chitosan acetate and 17\% EDTA might be attributed to that, dentin is much more sclerosed in the apical region with less content of collagen fibers to which AH Plus sealer bond ${ }^{(24)}$.

In group III $(\mathrm{NaOCl})$, the highest mean push out bond strength was recorded at the apical level compared to the coronal and middle levels with no statistical significant difference among the root levels. This could be attributed to the low viscosity of $\mathrm{NaOCl}$ which allow it to reach the apical level and act on dentin surface as a fat and organic solvent which degrade fatty acids, converting them into glycerol (alcohol) and salts (soap), that decrease the surface tension of the remaining solution, which improve $\mathrm{NaOCl}$ wettability to the treated dentin surface, therefore it may enhance AH Plus sealer's wettability to radicular dentin ${ }^{(25)}$. These results are consent with a previous study, which revealed that the greatest mean push out bond strength of AH plus sealer was recorded at the apical level compared to coronal and middle levels in case of presence of smear layer ${ }^{(26)}$. 
Regarding SEM observation of sealer/ dentin interface of tested samples which were treated with $\mathrm{NaOCl}$ (group III) revealed that there was no penetration of sealer into dentinal tubules at coronal third. However, at the middle third there was minimal sealer penetration. This could be attributed to the fact that, when the root canal obturated with lateral condensation technique, finger spreader and gutta-percha cones exert pressure that is directed to the root canal walls resulting in lateral forces especially at middle third which favored sealer penetration into dentinal tubules ${ }^{(27)}$. This finding was correlated with another study which reported that $\mathrm{AH}$ plus sealer penetration into dentinal tubules was noticed when $\mathrm{NaOCl}$ used alone to irrigate the root canal without any chelating agent ${ }^{(28)}$. In contrary, a previous study reported that penetration of $\mathrm{AH}$ plus sealer was observed only in case of removing smear layer by $17 \%$ EDTA and was not observed in case of using $\mathrm{NaOCl}$ alone without chelating agent ${ }^{(29)}$. These controversial results could be attributed to using various volume and concentration of $\mathrm{NaOCl}$ irrigating solution following the use of each file.

In the current study, the features noticed in the SEM assessment of the sealer/ dentin interface regarding sealer penetration into the dentinal tubules and tags formation did not show correlation with the push out bond strength evaluation. This could be attributed to that the resin component of resinbased sealers penetrates dentinal tubules, whereas filler particles remain at the interface because they are too large to penetrate the tubules. Resin depletion from the interfacial layer may be the cause of the low push-out bond strength ${ }^{(30)}$. This finding approves the hypothesis that the penetration of resin tags into dentinal tubules is not the most effective factor influencing the sealers' adhesion to radicular dentin. Adhesion process relay on several chemical and physical aspects between dentinal walls and the materials ${ }^{(31)}$.

These results are in consent with a previous study which studied the adhesion of endodontic sealers and found that removal of smear layer was not associated with higher bond strength although penetration of sealer into the dentinal tubules ${ }^{(32)}$. In contrary, the results of the current study are not consent with other studies which indicate a direct relationship between the sealers penetration into the tubules and values of the push out bond strength ${ }^{(30,33)}$.

Regarding overall comparison between groups, the results showed that, using $0.2 \%$ chitosan acetate and $17 \%$ EDTA as final irrigating solution (group I \& II respectively) decreased the push out bond strength of $\mathrm{AH}$ plus sealer to radicular dentin compared to $\mathrm{NaOCl}$ alone without chelating agent (group III). The possible explanation for the reduction of push out bond strength may be that opened dentinal tubules act as stress risers, which promoted failure in the adhesive joint. These failures result from locally high stresses in specific areas and contribute to the propagation of adhesive failure ${ }^{(15)}$.

The results of this study are correlated with recent studies which reported that the bond strength of $\mathrm{AH}$ plus sealer to radicular dentin reduced by using 17\% EDTA as final irrigating solution ${ }^{(14,15)}$. On the contrary, another study reported that the bond strength of AH Plus sealer to radicular dentin improved upon using of $0.2 \%$ chitosan acetate and $17 \%$ EDTA as final irrigating solutions ${ }^{(13)}$. This could be attributed to using different smear layer removing protocol as they immersed the samples in $5.25 \% \mathrm{NaOCl}$ solution then immersed them in either $17 \%$ EDTA solution or $0.2 \%$ chitosan solution.

The results revealed that, a statistically significant difference in the mean push out bond strength among group III $(\mathrm{NaOCl})$ and control group (saline) was present, where the samples treated with $\mathrm{NaOCl}$ showed higher values. This finding was in consent with a previous study which concluded that the push-out bond strength of $\mathrm{AH}$ plus sealer when the specimens irrigated with $\mathrm{NaOCl}$ was higher than when irrigated with saline ${ }^{(34)}$. 


\section{CONCLUSION}

The following could be concluded within the limitations of this study:

1. Chitosan acetate $0.2 \%$ as natural product attracts its use as dentin chelating agent.

2. The effect of $0.2 \%$ chitosan acetate on the push out bond strength of AH Plus sealer was comparable to that of $17 \%$ EDTA when used as a final rinse.

3. AH Plus sealer showed better adhesion to the dentin in the presence of smear layer especially at the apical third.

Evaluation of the impact of using $0.2 \%$ chitosan acetate as a final rinse on the push out bond strength of other sealers to the radicular dentin is recommended.

\section{REFERENCES}

1. Peters OA, Schonenberger K, Laib A. Effects of four Ni-Ti preparation techniques on root canal geometry assessed by micro computed tomography. Int Endod J 2001; 34: 22130 .

2. Parente JM, Loushine RJ,Susin L. Root canal debridement using manual dynamic agitation or the EndoVac for final irrigation in a closed system and an open system. Int Endod J 2010; 43: 1001-12.

3. Mozayeni MA, Zadeh YM, Paymanpour P, Ashraf H, Mozayani M. Evaluation of push-out bond strength of AH26 sealer using MTAD and combination of $\mathrm{NaOCl}$ and EDTA as final irrigation. Dent Res J 2013; 10: 359-63.

4. Jaju S and Prashant P. Newer Root Canal Irrigants in Horizon. Int J Dent 2011, 2011:1-9.

5. Kurita K. Chemistry and application of chitin and chitosan. Polymer Degradation and Stability 1998; 59: 117-20.

6. Jeon YJ, Shahid F, Kin SK. Preparation of chitin and chitosan oligomers and their applications in physiological functional foods. Food Reviews International 2000; 16: 159-76.

7. Fawzy MI. Scanning electron microscopic evaluation of the effectiveness of chitosan solution in smear layer removal. Egy Dent J 2011; 57:419-24.
8. Silva PV, Guedes DF, Pécora JD, Cruz-Filho AM. Timedependent effects of chitosan on dentin structures. Braz Dent J 2012; 23: 357-61.

9. Kamble AB, Abraham S, and Mehta DL. Scanning electron microscopic evaluation of efficacy of $17 \%$ ethylenediaminetet-raacetic acid and chitosan for smear layer removal with ultrasonics: an in vitro study. Contemp Clin Dent 2017; 8: 621-6.

10. Rahimi M, Jainaen A, Parashos P, Messer HH. Bonding of resin-based sealers to root dentine. J Endod 2009; 35:121-4.

11. Scha“fer E, Zandbiglari T, Scha“fer J. Influence of resinbased adhesive root canal fillings on the resistance to fracture of endodontically treated roots: an in vitro preliminary study. Oral Surg Oral Med Oral Pathol Oral Radiol Endod 2007 ;103: 274-9.

12. Shivanna V. The effect of different irrigating solutions on the push out bond strength of endodontic sealer to dentin and assessing the fracture modes: An In-vitro study. ICDRO J 2014; 6: 86-91.

13. Bayram HM, Bayram E, Kanber M, Celikten B, Saklar F. Effect of different chelating solutions on the push-out bond strength of various root canal sealers. Biomed Res India 2017; 401-6.

14. Adl AR, Sobhnamayan F, Ghanbaran S, Ahzan Sh. Effect of smear layer removal on bond strength of two different resin-based sealers; an in vitro study. JDB 2017; 4, 445-9.

15. Sheena P, Raj I, Ramesh R, Christalin R. Adhesion of epoxy resin based endodontic sealers to the root canal with and without smear layer removal. IOSR-JDMS 2017; 16:106-12.

16. Teixeira CS, Alfredo E, Thomé LHC, Gariba-Silva R, Silva-Sousa YTC and Sousa MD. Adhesion of an endodontic sealer to dentin and gutta percha: shear and push out bond strength measurements and SEM analysis. J Appl Oral Sci 2009; 17: 129-35.

17. Da cruz-Filho AM, De Vito Bordin AR, Souza-Flamini LE, Da costa Guedes DF, Saquy PC, et al. Analysis of the shelf life of chitosan stored in different types of packing, using colorimetry and dentine microhardness. Restor Dent Endod 2017; 42: 87-94.

18. Sundqvist G, Figdor D, Persson S, Sjo“gren U. Microbiologic analysis of teeth with failed endodontic treatment and the outcome of conservative re-treatment. Oral Surg Oral Med Oral Pathol Oral Radiol Endod 1998; 85: 86-93. 
19. Silveira LFM, SilveiraCF, Martos J, Suita de Castro LA. Evaluation of the different irrigation regimens with sodium hypochlorite and EDTA in removing the smear layer during root canal preparation. Journal of Microscopy and Ultrastructure 2013; 1: 51-6.

20. Violich and Chandler NP. The smear layer in endodontics - a review. Int Endod J 2010; 43: 2-15.

21. Jaju $S$ and Prashant P. Newer root ranal rrrigants in Horizon. Int J Dent 2011, ID 851359:1-9.

22. Mjor IA and Nordahl I. The density and branching of dentinal tubules in human teeth. Arch Oral Biol 1996;41: 401-12.

23. Haragushiku GA, Teixeira CS, Furuse AY, Sousa YTS, Neto MDS, and Silva RG. Analysis of the interface and bond strength of resin-based endodontic cements to root dentin. Microsc Res Tech 2012; 75:655-61.

24. Kim YK, Grandini S, Ames JM, Kim SK, Pashley DH, Gutmann JL, et al. Critical review on methacrylate resinbased root canal sealers. J Endod 2009; 36: 383-99.

25. Esterla C, Cyntia RA, Barbin EL. Mechanism of action of sodium hypochlorite. Braz Dent J 2002; 13:113-7.

26. Hamid HA and Al-Azzawi AJ. The effect of smear layer on push-out bond strength to dentin of Bioceramic sealer (In vitro study). J Bagh College Dentistry 2013; 25: 5-11.

27. Macedo LMD, Sousa YS, Silva SRC, Baratto SSP, Filho FB, Abi Rached FJ. Influence of root canal filling techniques on sealer penetration and bond strength to dentin. Braz Dent J 2017; 28: 380-4.
28. Sonu KR, Girish TN, Ponnappa KC, Kishan KV, Thameem PK. Comparative evaluation of dentinal penetration of three different endodontic sealers with and without smear layer removal" - Scanning electron microscopic study. Saudi Endod J 2016; 6: 16- 20.

29. Kokkas AB, Boutsioukis AC, Vassiliadis LP and Stavrianos CK. The influence of smear layer on dentinal tubule penetration depths by three different root canal sealers- an in vitro study. J Endod 2004; 30:100-2.

30. Jainaen A, Palamara JE, Messer HH. Push-out bond strengths of the dentine-sealer interface with and without a main cone. Int Endod J 2007; 40:882-90.

31. Oksan T, Aktener BO, Sen BH, Tezel H. The penetration of root canal sealers into dentinal tubules. A scanning electron microscopic study. Int Endod J 1993 26:301-5.

32. Saleh IM, Ruyter IE, Haapasalo MP, Orstavik D. Adhesion of endodontic sealers: scanning electron microscopy and energy dispersive spectroscopy. J Endod 2003; 29:595601.

33. Babb BR, Loushine RJ, Bryan TE, Ames JM, Causey MS, Kim J, et al. Bonding of self-adhesive (self-etching) root canal sealers to radicular dentin. J Endod 2009; 35: 578-82.

34. Ertas H and Sagsen B. Comparison of the effect of MTAD and conventional irrigation agents on apical leakage and push-out bond strength of root canal filling. Scaning 2015; 37: 393-8. 